\title{
SECONDARY COHOMOLOGY OPERATIONS AND COMPLEX VECTOR BUNDLES
}

S. GITLER, M. MAHOWALD AND R. JAMES MILGRAM ${ }^{1}$

Secondary cohomology operations have proved very useful in recent years [2], [5], [6].

Here we show that certain secondary operations are associated with the divisibility by 2 of the Chern classes of complex vector bundles, and so we obtain a very simple method for evaluating them.

Thus let $\Phi_{4 j}$ be the secondary operation based on the relation

$$
\mathrm{Sq}^{1} \mathrm{Sq}^{4 j}+\left(\mathrm{Sq}^{2} \mathrm{Sq}^{1}\right) \mathrm{Sq}^{4 j-2}+\mathrm{Sq}^{4 j} \mathrm{Sq}^{1}=0 .
$$

Let $e$ be a generator of $H^{2}\left(C P^{\infty}, Z\right)$ and $\rho: H^{*}(, Z) \rightarrow H^{*}\left(, Z_{2}\right)$ the coefficient homomorphism.

Theorem A. Let $k$ be divisible by 4 and suppose the 2jth integral Chern class $C_{2 j}$ of $k \eta$ ( $\eta$ is the canonical line bundle over $C P^{\infty}$ ) satisfies $C_{2 j}=2 \theta$; then $\Phi_{4 j}\left(\rho\left(e^{k}\right)\right)=\rho\left(e^{k}\right) \cup \rho(\theta)$ with zero indeterminacy.

The total Chern class of $k \eta$ is $(1+e)^{k}$; hence

$$
C_{2 j}=\left(\begin{array}{c}
k \\
2 j
\end{array}\right) e^{2 j}
$$

and we have for example

$$
\Phi_{2 r}\left(\rho\left(e^{2^{r}}\right)\right)=\rho\left(e^{2^{r}+2^{r-1}}\right) .
$$

More generally

Corollary. Let $i=2^{r} a$ and $4 j=2^{r-1} b$ with $a, b$ odd; then

$$
\left(\begin{array}{l}
2 a \\
b-1
\end{array}\right) \equiv 1(2)
$$

implies $\Phi_{4 j}\left(\rho\left(e^{i}\right)\right)=\rho\left(e^{i+2 j}\right)$.

This last result includes as special cases the basic results in this direction of [1], [5], [6]. The proof of A turns out to be very easy and is given in \$2. Further, our viewpoint simplifies the original proof of Hopf invariant one as given in [1] to the point where it may be even easier than the recent $K$-theory proofs of [3], [4], [7], so we include

Received by the editors March 25, 1968.

${ }^{1}$ This research supported in part by NSF Grant GP6560 and by the U. S. Army Research Office (Durham) for the last two authors. 
an outline proof in $\S 3$. Finally, in $\$ 4$ we extend these results to secondary operations on $R P^{\infty}$.

Theorem A is essentially 3.3.2 of [9] and the methods of proof are similar. A can also be obtained by using the results of [10] and $S$ duality $[11, \S 4]$. Maunder's approach allows one to evaluate certain higher order operations built up from the $\Phi_{4 j}$ in $C P^{\infty}$ as well. However our Theorem B does not seem to follow from [10] or [11], and the methods in $\$ \$ 2$ and 4 also generalize to higher order operations (see [8]). Indeed our main object in presenting this paper was to help clarify [8]. Moreover, the viewpoint on higher order operations implicit in this note seems to be lacking in the literature.

2. Theorem B and the proof of Theorem A. Let $E_{n}$ be the universal example for $\Phi_{4 j}$ on an integral class of dimension $n$. Thus $E_{n}$ is the fiber in the map

$$
\begin{aligned}
\theta & =\mathrm{Sq}^{4 j-2}(\iota) \times \mathrm{Sq}^{4 j}(\iota): K(Z, n) \\
& \rightarrow K\left(Z_{2}, n+4 j-2\right) \times K\left(Z_{2}, n+4 j\right)
\end{aligned}
$$

and we have the sequence of fibrations

$$
\begin{aligned}
F= & K\left(Z_{2}, n+4 j-3\right) \times K\left(Z_{2}, n+4 j-1\right) \stackrel{j}{\rightarrow} E_{n} \stackrel{\pi}{\rightarrow} K(Z, n) \\
& \stackrel{\theta}{\rightarrow} K\left(Z_{2}, n+4 j-2\right) \times K\left(Z_{2}, n+4 j\right) .
\end{aligned}
$$

Let $T(k)$ be the Thom space of the universal complex $k$-plane bundle, and denote the Thom class as $U \in H^{2 k}(T(k), Z)$. From the map $(U): T(k) \rightarrow K(Z, 2 k)$ and the fibration $\pi: E_{2 k} \rightarrow K(Z, 2 k)$ we have the induced fibration $(U)^{\sharp} E_{2 k}$ over $T(k)$ and the diagram of fibrations:

$$
\begin{array}{ll}
(U)^{\sharp} E_{2 k} & \underset{(\widetilde{U})}{\longrightarrow} E_{2 k} \\
\downarrow \tilde{\pi} & \\
T(k) \underset{(U)}{\longrightarrow} K(Z, 2 k)
\end{array}
$$

The following lemma is evident.

LEMmA 2.3. Let $v \in H^{*}\left(E_{2 k}\right)$ be a universal example for $\Phi_{4 j}$. Let $\bar{U}$ be the Thom class of a complex $k$-bundle $\xi$ over a space $X$; then

$$
\Phi_{4 j}(\bar{U})=\left\{L^{*}\left((\tilde{U})^{*} v\right)\right\}
$$

where $L$ runs over all liftings which make the following diagram com- 
mute:

$$
\begin{aligned}
&(U)+E_{2 k} \\
& L \nearrow \quad \downarrow \pi \\
& T(\xi) \rightarrow T(k)
\end{aligned}
$$

LEMMA 2.4. In $H^{*}\left(U^{*} E_{2 k}, Z\right)$ there are classes $X, Y$ so

(i) $\tilde{\pi}^{*}\left(C_{2 j-1} \cup U\right)=2 X$

(ii) $\tilde{\pi}^{*}\left(C_{2 j} \cup U\right)=2 Y$

(iii) $\tilde{U}^{*}(\mu)=\rho(Y)+\operatorname{Sq}^{2} \rho(X)+\tilde{\pi}^{*}(\rho(P \cup U))$ where $P$ is a polynomial of degree $4 j$ in the Chern classes $C_{1}, \cdots, C_{2 j-2}$,

Proof. $H^{2 k+4 j}\left(U^{*} E_{2 k}, Z_{2}\right)=\left(\operatorname{im} \tilde{\pi}^{*}\right) \oplus\left(\mathrm{im} j^{*}\right)$, and also $j^{*}\left(\tilde{U}^{*}(v)\right)$ $=\mathrm{Sq}^{1}\left(\iota_{2 k+4 j-1}\right)+\mathrm{Sq}^{2} \mathrm{Sq}^{1}\left(\iota_{2 k+4 j-3}\right)$; hence the lemma follows when we observe that if $X, Y$ exist then they must restrict to $\mathrm{Sq}^{1}\left(\iota_{2 k+4 j-8}\right)$, $\mathrm{Sq}^{1}\left(\iota_{2 k+4 j-1}\right)$ respectively, since this is true in the universal example for division by two, namely the fibering

$$
K\left(Z_{2}, n-1\right) \rightarrow K(Z, n) \stackrel{2(\iota)}{\longrightarrow} K(Z, n) .
$$

On the other hand, to show the existence of $X, Y$ is now completely direct.

Putting these results together we have

TheOREM B. Let $B$ be the Thom space of $\xi$, a complex $k$-bundle over a space $X$ for which $\rho\left(C_{1}\right)=0$. Suppose there are integer classes $X, Y$ so $C_{2 j-1}(\xi)=2 X, C_{2 j}(\xi)=2 Y$; then $\Phi_{4 j}(\rho U)$ is defined in $H^{*}\left(T, Z_{2}\right)$ and modulo the total indeterminacy

$$
\Phi_{4 j}(\rho U)=\left[\operatorname{Sq}^{2} \rho(X)+\rho(Y)\right] \cup \rho(U) .
$$

To complete the proof of $B$ note that in the map

$$
\lambda: \Sigma^{2 k+4 j-4} T(2 j-2) \rightarrow T(k)
$$

we have

$$
\lambda^{*} \rho(P \cup U)=\Phi_{4 j}\left(\sigma^{2 k-4 j-4} U_{2 j-2}\right)=\sigma^{2 k-4 j-4} \Phi_{4 j}\left(U_{2 j-2}\right)
$$

and $v$ can be chosen so this last is zero, ${ }^{2}$ but since $P$ is a polynomial in $C_{1}, \cdots, C_{2 j-2}$ the fact that $\lambda^{*}(\rho P \cup U)=0$ implies $\rho(P \cup U)=0$ and B follows since $\operatorname{Sq}^{2}(\rho(U))=0$.

To complete the proof of A note that the Thom space of $k \eta$ over

\footnotetext{
${ }^{2} \Phi_{4 j}$ is defined on $\rho$ of any integral $4 j-3$ class, and thus its value on a $4 j-4$ class $a$ is a stable cohomology operation $\operatorname{Sq}^{I}(a)$. Then we choose a new representant for $\Phi$, specifically we set $v^{\prime \prime}=v+\mathrm{Sq}^{\prime}(\iota)$.
} 
$C P^{\infty}$ is $C P^{\infty} / C P^{k-1}$. The theorem now follows from $\mathrm{B}$ and the evident map $p: C P^{\infty} \rightarrow C P^{\infty} / C P^{k-1}$ which collapses the $2 k-1$ skeleton to the base point since $\rho\left(p^{*} T(k \eta)^{*}(U)\right)=\rho\left(e^{k}\right)$.

3. Hopf invariant one. $Q(2)$ is the Steenrod algebra [12]. $a(2)$ $\otimes a(2)$ becomes an $a(2)$ module when we set $\alpha(a \otimes b)=\alpha a \otimes b$, and $m: a(2) \otimes a(2) \rightarrow a(2)$ is given by $m(a \otimes b)=a b$.

Definition 3.1. An element $x=\sum a_{i} \otimes S q^{2^{i}} \in Q(2) \otimes Q(2)$ is a minimal relation if $m(x)=0$.

Clearly the set of minimal relations is a module $g$ over $Q(2)$ and a basic set of relations is any $a(2)$ basis for $\mathscr{g}$, say $\left\{e_{i}: i \in I\right\}$ for some index set $I$. A relation among relations is an element $y=\sum a_{i} \otimes b_{i, j}$ $\otimes \mathrm{Sq}^{2^{j}}$ in $Q(2) \otimes Q(2) \otimes Q(2)$ so $(m \otimes 1)(y)=(1 \otimes m)(y)=0$.

Lemma 3.2. Let $E_{n, i}$ be the two-stage Postnikov system obtained by killing $\mathrm{Sq}^{1}(\iota), \mathrm{Sq}^{2}(\iota), \mathrm{Sq}^{4}(\iota) \cdots \mathrm{Sq}^{2^{i}}(\iota)$ in $H^{*}\left(K\left(Z_{2}, n\right), Z_{2}\right),\left(n>2^{k}\right)$; then $H^{*}\left(E_{n, i}, Z_{2}\right)$ is isomorphic to the module of minimal relations (over $Q(2)$ and with degree diminished by one) in dimension less than $n+2^{i+1}$. In dimension $n+2^{i+1}$ there is also $\mathrm{Sq}^{2^{i+1}}(\iota)$, and if $y$ is a relation among relations $\left(\sum a_{i} \otimes b_{i, j} \otimes \mathrm{Sq}^{2^{j}}\right)$ then in $H^{*}\left(E_{n, i}, Z_{2}\right)$ we have $\sum a_{i}\left\{b_{i, j} \otimes S_{2}^{2 j}\right\}=\lambda(y) \operatorname{Sq}^{2^{i+1}}(\iota)$ where $\lambda=0$ or 1 and depends only on $y$.

(The proof is a simple exercise with the Serre spectral sequence, the Steenrod algebra and the stability of the Steenrod operations.)

Lemma 3.3 (J. F. Adams). (a) A basic set of minimal relations is given by a doubly indexed family $R(i, j)$ (of degree $\left.2^{i}+2^{j}\right) 0 \leqq i<j-1$ or $i=j$. (b) There is a relation among relations of the form

$$
\left(\mathrm{Sq}^{2 i}+b\right)\{R(0, i)\}+\cdots
$$

where $b$ is decomposable, for $i \geqq 3$.

(The proof is an exercise in handling $a(2)$. Part (b) follows, for example, by proving $h_{0} h_{i}^{2}$ is nonzero in $\operatorname{Ext}_{\alpha(2)}\left(Z_{2}, Z_{2}\right)$ for $i \geqq 3$.)

Now consider the mapping $(\iota): E_{n, i} \rightarrow E_{n}$ where $E_{n}$ is the universal example for $\Phi_{2}{ }^{i}$. The following is immediate.

Lemma 3.4. ( ()$^{*}(v)=\{R(0, i)\}+\sum a_{j, k}\left\{R_{(j, k)}\right\}$ where $k<i$.

Now the basic theorem of [1] becomes

TheOREM 3.5. In $H^{*}\left(E_{n, i}, Z_{2}\right)$ we have $\left(\mathrm{Sq}^{2^{i}}+b\right)\{R(0, i)\}+\cdots$ $=\mathrm{Sq}^{2^{i+1}}(\iota)$ for $i \geqq 3$, and thus there is no two cell complex with $\mathrm{Sq}^{2^{i+1}}$ nonzero, $i \geqq 3$.

Proof. By A and 3.4 the only secondary operation among the 
$\{R(j, k)\}(k \leqq i)$ which is nonzero on $\rho\left(e^{2^{i}}\right)$ is $\{R(0, i)\}$ (the $R(j, k)$ for $j>0$ all have odd degree, and $R(0, k)$ for $k<i$ are 0 by A). Hence $\left[\left(\mathrm{Sq}^{2^{i}}+b\right)\{R(0, i)\}+\cdots\right]\left(\rho\left(e^{2^{i}}\right)\right)=\operatorname{Sq}^{2^{i}}\{R(0, i)\} \rho\left(e^{2^{i}}\right)=\mathrm{Sq}^{2^{i}} \rho\left(e^{2^{i}+2^{i-1}}\right)$ $=\mathrm{Sq}^{2^{i+1}}\left(\rho\left(e^{2^{i}}\right)\right)$ and the proof is complete.

4. Secondary operations in $R P^{\infty}$. Consider again the results of $\S 3$. $\Phi_{8}$ is defined and nonzero on $\rho\left(e^{8+16 k}\right)$ in $H^{*}\left(C P^{\infty}\right)$. But if $\pi: R P^{\infty}$ $\rightarrow C P^{\infty}$ is the nontrivial map then $\Phi_{8}\left(\pi^{*}\left(\rho\left(e^{8+16 k}\right)\right)\right)=0$ due to indeterminacy. On the other hand, from the universal relation $\mathrm{Sq}^{16}$ $=\mathrm{Sq}^{8} \Phi_{8}+\cdots$ it follows that the set

$$
\left\{\Phi_{8}\left(\pi^{*}\left(\rho\left(e^{8+16 k}\right)\right)\right),\{R(3,3)\}\left(\pi^{*}\left(\rho\left(e^{8+16 k}\right)\right)\right), \cdots\right\}
$$

cannot all vanish simultaneously. Thus this set of operations, even modulo indeterminacy, is not zero on the class of dimension $16+32 k$ in $H^{*}\left(R P^{\infty}, Z_{2}\right)$.

There are similar results with $\mathrm{Sq}^{16}$ replaced by $\mathrm{Sq}^{2^{i}}(i>4)$, and we have

TheOREM 4.1. There is no map

$$
R P^{2^{i+1}(k+1)-1} 2^{i}+2^{i+1} k \rightarrow S^{2^{i}+2^{i+1} k}
$$

inducing the nontrivial map in cohomology ( $R P^{s}$ is $R P^{s}$ with the $t-1$ skeleton collapsed to the base point).

Actually we can sharpen this result. Consider, for example, the pair of relations

$$
\begin{array}{r}
\mathrm{Sq}^{1} \mathrm{Sq}^{8 k}+\left(\mathrm{Sq}^{2} \mathrm{Sq}^{1}\right) \mathrm{Sq}^{8 k-2}+\mathrm{Sq}^{8 k} \mathrm{Sq}^{1}=0 \\
\mathrm{Sq}^{2} \mathrm{Sq}^{8 k}+\mathrm{Sq}^{4} \mathrm{Sq}^{8 k-2}+\mathrm{Sq}^{8 k} \mathrm{Sq}^{2}+\mathrm{Sq}^{8 k+1} \mathrm{Sq}^{1}=0
\end{array}
$$

Let $G_{n}$ be the universal example for both operations, let $u \in H^{*}\left(G_{n}\right)$ represent $\Phi_{8 k}$ and $\omega$ represent the second operation; then

$$
\begin{gathered}
4.2=\left(\mathrm{Sq}^{9}+\mathrm{Sq}^{6} \mathrm{Sq}^{2} \mathrm{Sq}^{1}\right) A+\mathrm{Sq}^{10} \mathrm{Sq}^{1} B+\left(\mathrm{Sq}^{7}+\mathrm{Sq}^{4} \mathrm{Sq}^{2} \mathrm{Sq}^{1}\right) \mathrm{Sq}^{8 k} C \\
+\left(\mathrm{Sq}^{8}+\mathrm{Sq}^{7} \mathrm{Sq}^{1}\right) \mathrm{Sq}^{8 k} D
\end{gathered}
$$

where $D$ is the $n$-dimensional generator on the fiber, $C$ is the $n+1$ dimensional generator, etc.

Let $\rho\left(e^{t}\right)$ satisfy $\Phi_{8 k}\left(\rho\left(e^{t}\right)\right) \neq 0$ in $H^{*}\left(C P^{\infty}\right)$; then there is a map $m: C P^{\infty} \rightarrow G_{2 t}$, and we have $m^{*}(\iota)=\rho\left(e^{t}\right) m^{*}(u) \neq 0$ and $m^{*}(\omega)=0$. Thus the same is true for $m \pi: R P^{\infty} \rightarrow G_{2 t}$. Moreover, any two liftings 


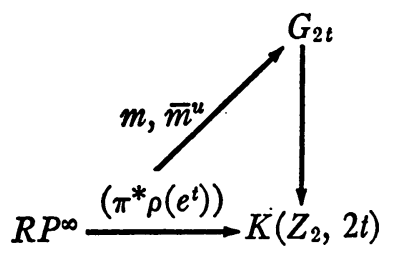

differ by a map into the fiber $F$. Thus if there were a lifting $\bar{m}$ so $\bar{m}^{*}(u)=\bar{m}^{*}(\omega)=0$ there would be a map $r: R P^{\infty} \rightarrow F$ and

$$
\text { 4.3. } \quad r^{*}\left(j^{*}(u)\right) \neq 0 \quad \text { while } r^{*}\left(j^{*}(\omega)\right)=0 \text {. }
$$

Theorem 4.4. Suppose $k=1+4 s$. Then $\left(\Phi_{8 k} ; \omega\right)$ on $\left(\pi^{*} \rho\left(e^{8(1+2 \lambda)}\right)\right)$ cannot both vanish simultaneously if

$$
\left(\begin{array}{l}
\lambda \\
s
\end{array}\right) \neq 0 .
$$

Proof. Under these assumptions

$$
\begin{aligned}
& \mathrm{Sq}^{8 k} \rho\left(e^{8(1+2 \lambda)+e}\right)=0 \quad(\epsilon=0,1), \\
& \Phi_{8 k}\left(\rho\left(e^{8(1+2 \lambda)}\right)\right) \neq 0, \\
& \mathrm{Sq}^{8} \Phi_{8 k} \rho\left(e^{8(1+2 \lambda)}\right) \neq 0 .
\end{aligned}
$$

Suppose now there were a map $r: R P^{\infty} \rightarrow F$ satisfying 4.3 ; then from 4.2

$$
r^{*}\left(\mathrm{Sq}^{8} j^{*}(u)+\mathrm{Sq}^{2}+\mathrm{Sq}^{4} \mathrm{Sq}^{2} \mathrm{Sq}^{1} j^{*}(\omega)\right)=0
$$

but this is impossible since $\operatorname{Sq}^{8}\left(r^{*} j^{*}(u)\right) \neq 0$ and by assumption $r^{*} j^{*}(\omega)=0$.

Similar results can be obtained for $\Phi_{8 k+4}$ but we need three operations based on

$$
\begin{array}{r}
\mathrm{Sq}^{1} \mathrm{Sq}^{8 k+4}+\left(\mathrm{Sq}^{2} \mathrm{Sq}^{1}\right) \mathrm{Sq}^{8 k+2}+\mathrm{Sq}^{8 k+4} \mathrm{Sq}^{1}=0 \\
\mathrm{Sq}^{4} \mathrm{Sq}^{8 k+2}+\mathrm{Sq}^{8 k+4} \mathrm{Sq}^{2}=0 \\
\mathrm{Sq}^{4} \mathrm{Sq}^{8 k+4}+\mathrm{Sq}^{8 k+6} \mathrm{Sq}^{2}+\mathrm{Sq}^{8 k+7} \mathrm{Sq}^{1}=0
\end{array}
$$

and if $u$ represents the first, $\omega$ the second, and $\chi$ the third, we look at

$$
j^{*}\left(\mathrm{Sq}^{8}(u)+\mathrm{Sq}^{4} \mathrm{Sq}^{2} \mathrm{Sq}^{1}(\bar{\omega})+\mathrm{Sq}^{2} \mathrm{Sq}^{1} \mathrm{Sq}^{2}(\chi)\right)
$$

on the fiber. The reader can easily supply details and further generalizations.

REMARK. One could, of course, verify directly (as in [2], [5], [6]) that these operations do not vanish simultaneously, since we know 
how to evaluate the indeterminacy. However, the method given here generalizes to higher order operations as in $\$ 3.4$ of [8].

\section{REFERENCES}

1. J. F. Adams, On the non-existence of elements of Hopf invariant one, Ann. of Math. 72 (1960), 20-104.

2. —-, Vector fields on spheres, Topology 1 (1962), 63-65.

3. - On the groups $J(X)$. IV, Topology 5 (1966), 21-71.

4. J. F. Adams and M. F. Atiyah, K-theory and the Hopf invariant, Quart. J. Math. 17 (1966), 31-38.

5. J. Adem and S. Gitler, Secondary characteristic classes and the immersion problem, Boll. Soc. Mat. Mexicana (2) 8 (1963), 53-78.

6. - Non-immersion theorems for real projective spaces, Boll. Soc. Mat. Mexicana (2) 9 (1964), 37-50.

7. E. Dyer, Chern characters of certain complexes, Math. Z. 80 (1963), 363-373.

8. S. Gitler, M. Mahowald and R. J. Milgram, The non-immersion problem for $R P^{n}$ and higher order cohomology operations, Proc. Nat. Acad. Sci. 60 (1968), 432-437.

9. M. Mahowald and F. Peterson, Secondary cohomology operations on the Thom class, Topology 2 (1963), 367-377.

10. C. R. F. Maunder, Chern characters and higher order cohomology operations, Proc. Cambridge Philos. Soc. 60 (1964), 751-764.

11. - Cohomology operations of the Nth kind, Proc. London Math. Soc. (3) 13 (1963), 125-154.

12. N. Steenrod and D. B. A. Epstein, Cohomology operations, Princeton Univ. Press, Princeton, N. J., 1962.

Centro de Investigacion del I. P. N., NORTHWESTERN UNIVERSITY, AND University of Illinois at Chicago Circle 